\title{
Regrowth of amorphous films
}

\author{
S. S. Lau \\ California Institute of Technology, Pasadena, California 91125 \\ (Received 14 August 1978; accepted 16 August 1978)
}

In this review, we emphasize three aspects of the regrowth of ion-implanted amorphous $\mathrm{Si}$ layers: (1) orientation dependence of the regrowth kinetics, (2) impurity effects on the regrowth kinetics, and (3) impurity distribution due to regrowth. To account for the orientation dependence there are at least three proposed models: (1) geometric model, (2) stress relaxant model, and (3) surface reconstruction model. Each of these models is discussed here. For amorphous Ge regrowth, the characteristics are similar to those of Si. Parallels are drawn whenever possible. An example is given to illustrate the use of ion-implanted-regrowth process to modify the crystallinity of thin layers.

PACS numbers: $68.55 .+\mathrm{b}, 61.70 . \mathrm{Tm}$

\section{INTRODUCTION}

The recrystallization of amorphous films (semiconductor materials and others) is a vast area of scientific and practical interest. In the discussion of this subject here, we restrict the discussion to the epitaxial regrowth (or recrystallization) of amorphous semiconductor, in particular, $\mathrm{Si}$ and Ge layers.

The simplest system, in concept, is that of an amorphous layer deposited directly on a single-crystal substrate. However, unless stringent surface cleaning precautions are taken and the depositions carried out in ultrahigh vacuum, ${ }^{1}$ the presence of surface oxide or impurity layers on the crystal surface prevent direct contact between the amorphous layer and substrate. This interfacial layer between the amorphous and single crystal regions blocks epitaxial growth during thermal annealing. This difficulty, however, can be surmounted by laser or electron beam annealing methods. ${ }^{\text {la }}$

Ion-implantation tehcniques can be used to obtain amorphous layers in direct contact with the single-crystal substrate that are virtually free of contamination. Generally speaking, a first ion-implantation step with self ions (i.e., $\mathrm{Si}$ into $\mathrm{Si}$ ) is used to create an amorphous layer that is free of impurities. A second implantation step can be used to introduce a known amount of impurities, if impurity effects are of interest. Concentrations of impurities of about 0.1 at. \% as well as the substrate orientation can have major influence on the regrowth kinetics. These results have been reported in the literature in the past and reviewed recently. ${ }^{2}$ An attempt will be made here to summarize recent results and proposed models on impurity (As, B, O, Ar, etc.) and orientation effects on growth kinetics. These effects are, perhaps, more pertinent to practical applications of regrowth of ion-implanted amorphous layers.

\section{THERMAL ANNEALING OF ION-IMPLANTED AMORPHOUS LAYERS}

In use of implantation techniques, there are several precautions that should be taken in regard to ion species, ion energies and substrate temperature. The dose of ions required is equivalent to about one monolayer $\left(\sim 10^{15} \mathrm{ions} / \mathrm{cm}^{2}\right)$ dis- tributed over the ion range $R_{p}$ (where $R_{p}$ is between 1000 and $4000 \AA$ ). It is also necessary to maintain ion species purity during implantation such that impurities with the same charge to mass ratio are absent in the beam. The thickness of the amorphous layer is somewhat greater than $R_{p}$ and depends on ion species and energy. To form a continuous amorphous layer from the surface to a depth $R_{p}$, multiple energy implantation procedures are usually used. The maintenance of low substrate temperature (near $\mathrm{LN}_{2}$ ) is also advisable to avoid beam annealing effects during implantation. Under proper conditions, it is possible to form approximately $0.5 \mu \mathrm{m}$ thick, impurity-free amorphous layer in direct contact with a single crystal substrate with implantation energies up to about 250 $\mathrm{keV}$.

\section{A. Orientation effects \\ 1. General phenomena}

The growth of epitaxial Si or Si is strongly dependent on the orientation of the underlying substrate. ${ }^{3}$ This is illustrated in Fig. 1 which shows regrown layer thickness vs annealing time at $550^{\circ} \mathrm{C}$ for $\langle 100\rangle,\langle 110\rangle$, and $\langle 111\rangle$ oriented Si substrates. The growth rate $(\sim 90 \AA / \mathrm{min})$ for $\langle 100\rangle$ samples is about three times that for $\langle 110\rangle$ samples and about 25 times the initial growth rate for $\langle 111\rangle$ samples. The growth for $\langle 100\rangle$ and $\langle 110\rangle$ is linear in time while the growth for $\langle 111\rangle$ is not.

The same orientation effects are found in Ge samples as in Si samples. In $\langle 100\rangle$ Ge samples, a growth rate of $\approx 90 \AA / \mathrm{min}$ is found at an annealing temperature around $350^{\circ} \mathrm{C}$. These temperatures in degrees Kelvin are close to half the melting point $\mathrm{Tm}$, of $\mathrm{Ge}$ and $\mathrm{Si}$. For both semiconductors, the growth can be described by an Arrenhius equation with an activation energy of $2.4 \mathrm{eV}$ for $\mathrm{Si}$ and 2.0 for Ge as shown in Fig. 2 for various orientations. ${ }^{3}$

The regrowth rate of $\mathrm{Si}$ as a function of substrate orientation is shown in Fig. $3 .^{3}$ For samples whose orientations are within $20^{\circ}$ of $\langle 100\rangle$ and $\langle 110\rangle$ directions the regrowth rates are linear with time. For samples near the $\langle 111\rangle$ orientation the measurements shown in Fig. 3 represent the regrowth rate over the first $1000 \AA$. 


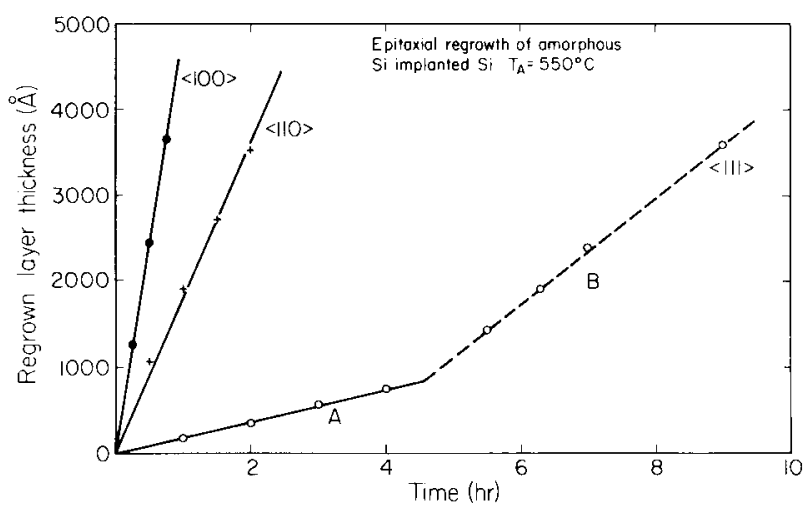

FIG. 1. Regrowth rates vs time for ${ }^{28} \mathrm{Si}$-implanted $\mathrm{Si}$ annealed at $550^{\circ} \mathrm{C}$ (taken from Ref. 2 and 3).

In transmission electron microscopy ${ }^{4,5}$ studies of the orientation dependence of growth, it was found that planar defects (twins and stacking faults) were dominant in samples orientated $16^{\circ}$ of the $\langle 111\rangle$ direction and dislocations were dominant in other orientations. Any proposed model for the regrowth phenomena must account for all these experimental observations.

\section{Models for orientations dependence growth}

At present there are at least three proposed models for the orientation dependence of growth. They are summarized briefly as follows:

(1) Geometric model ${ }^{3}$ : This model assumes no surface reconstruction at the amorphous-crystalline interface and that atoms can be transferred from amorphous to crystalline phase on sites where at least two nearest neighboring atoms at the interface are already in crystalline positions. This requirement leads to growth along (111) planes in the diamond lattice. An example is shown in Fig. 4 where the interface is parallel to

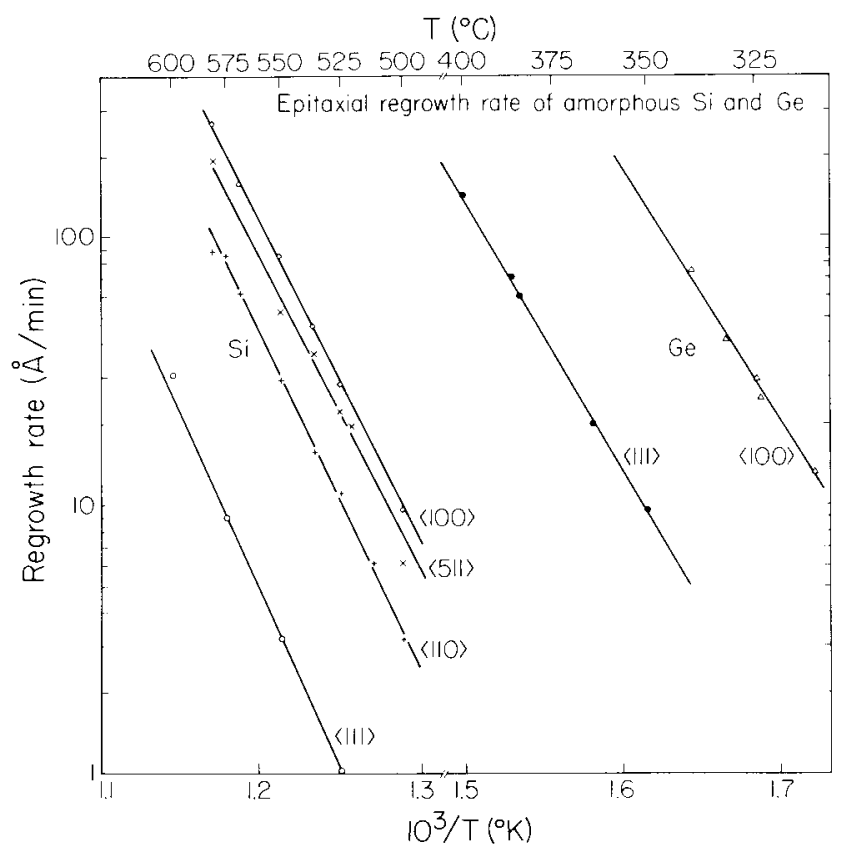

FIG. 2. Regrowth rate vs $10^{3} T^{-1}(\mathrm{~K})$ for different substrate orientations of Si and Ge (taken from Ref. 3).

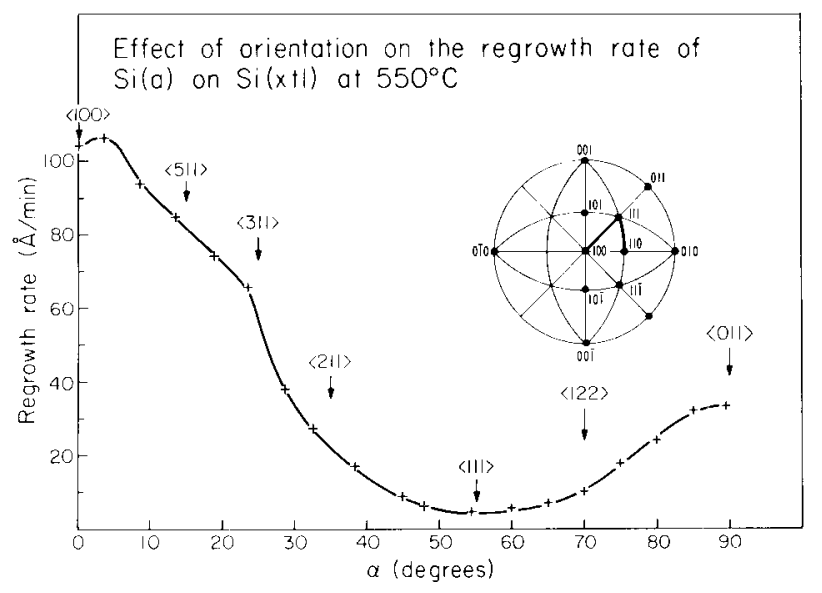

Fig. 3. Plot of regrowth rate at $550^{\circ} \mathrm{C}$ vs substrate orientation, for a series of samples cut along the direction indicated by the heavy lines in the standard triangle (taken from Ref. 3).

the (311) plane. The plane of the paper is the $(0 \overline{1} 1)$ plane and the atoms are aligned along the $[0 \overline{1} 1]$ direction. To satisfy the two-atom-in position criterion, the atoms marked $A$ will first recrystallize because the two atoms directly underneath it (in the $[\overline{\mathbf{1}} 00]$ direction) are in the crystalline phase along the $[0 \overline{1} 1]$ direction, then followed by the $B$ and $C$ atoms. As shown in Fig. 4 , the atom sequence follows the $[\overline{2} 11]$ direction on the (111) plane.

This is essentially a geometric argument which predicts an orientation dependence shown by the dashed line in Fig. 5. The dashed line was normalized to the value measured along the $\langle 100\rangle$ and is in qualitative agreement with experimental results up to the $\langle 111\rangle$ orientation. The predicted growth rate for $\langle 111\rangle$ oriented samples is zero, and it is speculated that the measured growth rate is due to nucleation of islands which lead to faults and twins. Growth of twinned regions on $\{111\}$ planes inclined to the surface could then lead to an irregular interface and nonconstant growth rate. TEM studies suggest that the initial growth rates correspond to the nucleation and growth of small twins and the later growth rates (after about $\sim 1000$ Å of growth) correspond to an average rate of regions

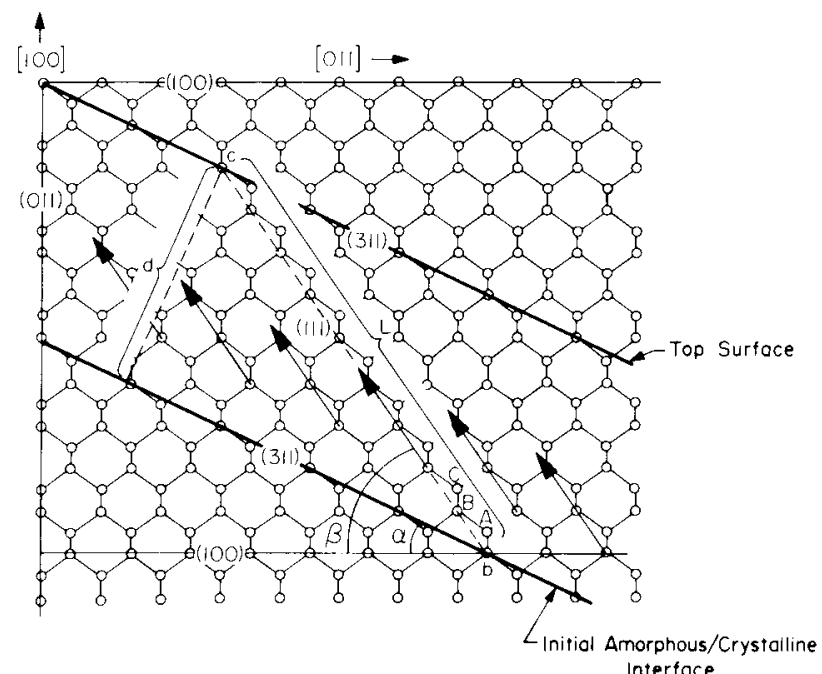

FIG 4. Section looking down the $[0 \overline{1} 1]$ axis in Si. See text for details (taken from Ref. 3) 


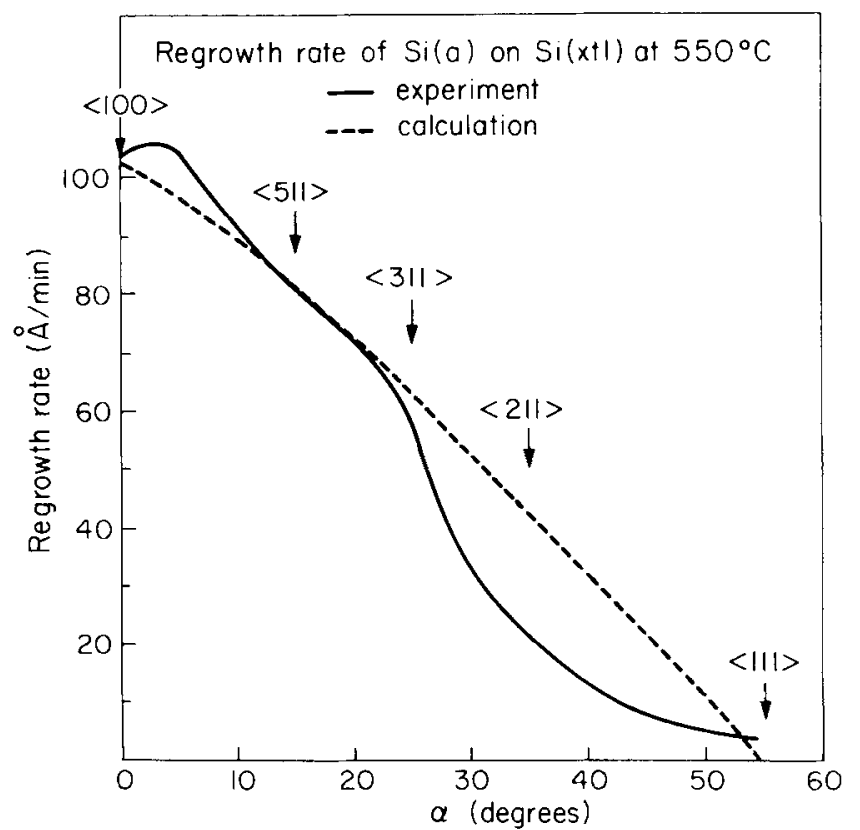

FIG. 5. Plot of regrowth rate at $550^{\circ} \mathrm{C}$ vs substrate orientation angle from the $\langle 100\rangle$ direction, from experiment (solid line) and from calculation (dashed line) (taken from Ref. 3).

containing relatively large twins. Since the orientation of the twins is $\langle 511\rangle$, one might expect the growth rate of such regions to be higher.

The major drawback of this model is that the model breaks down for orientations making an angle larger than $54.74^{\circ}$ with the (100) plane. For those orientations, the two-atom-in position criterion can no longer be satisfied.

(2) Stress relaxation model6: This model rationalizes the orientation dependence in terms of the difference in driving force for stress relaxation in the implanted layers. Ion-implanted layers are generally under a biaxial state of compressive stress. Recrystallization of the amorphous layer is expected to reduce the stresses greatly. This model assumes that stress relief occurs by the motion of Shockley partial dislocations $(b=1 / 6[211])$ under the influence of the resolved shear stresses on the (111) planes. The maximum shear stress in a plane inclined to the surface is in a direction perpendicular to the intersection of the inclined plane and the surface. For (111) planes inclined to (100), (110), and (111) surfaces this direction is a [112] direction while the intersection is a [110] direction. The maximum shear stress, $\tau$, on the (111) inclined to the surface by an angle $\theta$ is given by

$$
\tau=\sigma / 2 \sin 2 \theta
$$

where $\sigma$ is the biaxial stress in the implanted layer, and assumed to be independent of substrate orientation. The values of $\theta$ are $54.7^{\circ}, 35.3^{\circ}$, and $70.5^{\circ}$ and the factor $1 / 2 \sin 2 \theta$ is 0.47 , 0.47 , and 0.31 for $(100)$, (110), and (111) planes, respectively. Thus the maximum resolved shear stresses on (100) and (110) planes are 1.5 times larger than that on (111) planes and result in larger driving force to cause relaxation of stresses on these planes [Fig. 6(a)].

It is assumed that near the amorphous-crystalline interface, Frank partial dislocations with $\mathbf{b}=1 / 3[111]$ are nucleated upon heating by the aggregation of point defects in the crystalline region. The higher shear stresses on (100) and (110) samples enable the nucleation of $1 / 6$ [112] partial dislocation and under go the following interaction:

$$
1 / 3[111]+1 / 6[11 \overline{2}]=1 / 2[110] \text {. }
$$

The perfect dislocations $(b=1 / 2[110])$ intersecting the (100) and (110) surfaces provide abundant nucleation sites for

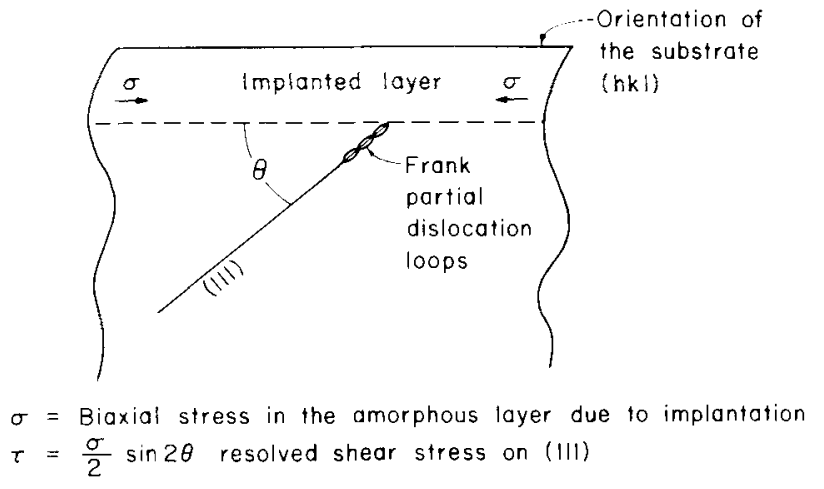

(a)

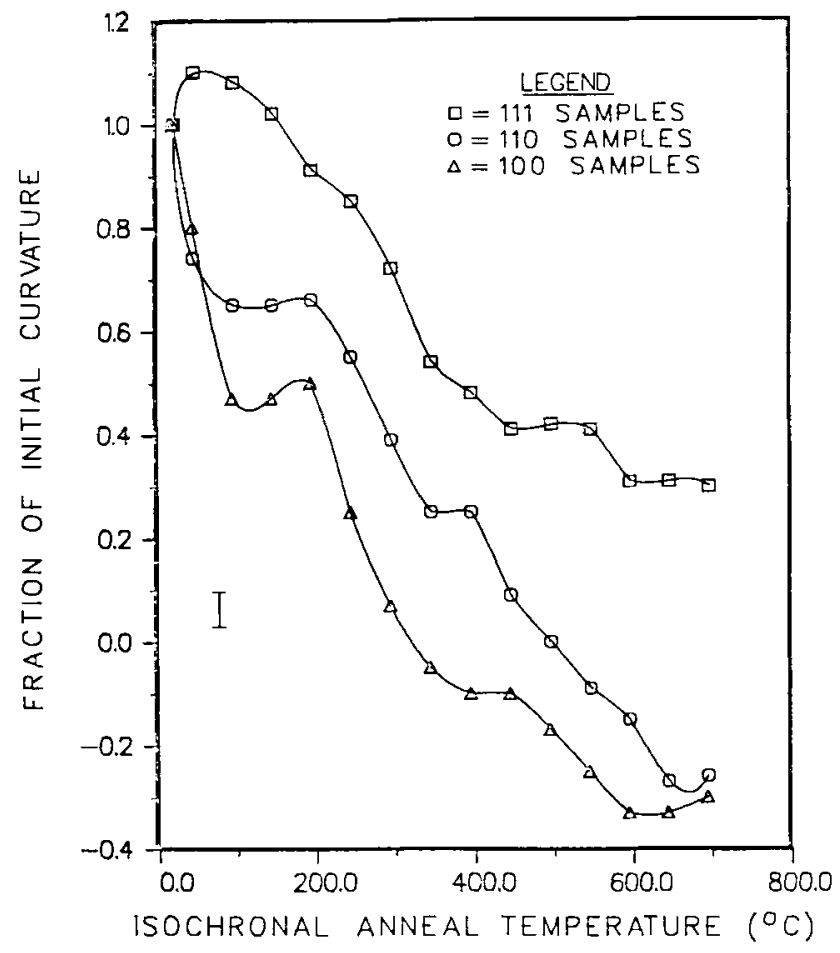

(b)

FIG. 6. (a) Stress relaxation model of regrowth (see text for details). (b) Relative amount of sample curvature, initially induced by stresses from a $4 \times 10^{15} \mathrm{~A}^{++}$ions $/ \mathrm{cm}^{2}$ implant, remaining after annealing vs 20 -min isochronal anneal temperature. Data points are average of data from two samples each of 111,110 , and 100 oriented samples. Error bar shows maximum scatter between two samples of a given orientation (taken from Ref. 6). 
growth. For (111) samples, the shear stresses are too low to unfault the Frank loops which do not provide ready nucleation sites for growth and therefore impede crystal growth.

There are two general drawbacks of this model. From the shear stress argument, the (100) and (110) samples should have the same regrowth rate, in obvious disagreement with experimental result. The stress relaxation measurements present in Fig. 6(b) showed that the stresses in (100) and (110) samples went from compressive to tensile between $400^{\circ}$ and $600^{\circ} \mathrm{C}$, the range of temperature where regrowth occurs. This observation is inconsistent with proposed stress relief mechanism, where little or no stress should have been observed for completely regrown layers.

(3) Surface reconstruction model 7 : This model assumes that the amorphous-crystalline interface reconstructs and accepts the fact that (100) samples regrow 25 times faster than the (111) samples. It argues that for (100) samples, the regrowth rate is so fast that reconstruction does not occur for this orientation and leads to little or no planar defects in the regrown layers. For (111) samples, the interface has enough time to relax into a reconstructed surface before the next layer of atoms grows on top of the reconstructed surface. Since the reconstructed surface is expected to be associated with excess surface vacancies, the buried vacancies give rise to the observed planar defects in (111) samples. This model is intended to account for different defect structures in the regrown layer and not the regrowth kinetics.

Judging from these models, there is still ample room for a more complete model to account for all experimental observations.

\section{B. Impurity effects on regrowth kinetics}

Implantation techniques also offer the possibility to incorporate known amounts of impurities in the implantedamorphous layer. The presence of these impurities can have a pronounced influence on the growth kinetics. ${ }^{8-10}$ Table I lists some representative values of the regrowth rate with various impurities. Of the species listed, ${ }^{11} \mathrm{~B}$ produces the strongest increase and ${ }^{40} \mathrm{Ar},{ }^{20} \mathrm{Ne}$, and ${ }^{16} \mathrm{O}$ produce the strongest decrease in growth rate.

Figure 7 shows an example of regrowth rates in ${ }^{31} \mathrm{P}$-implanted layers. ${ }^{9}$ At low concentrations $\left(\sim 0.5 \times 10^{20} \mathrm{~cm}^{-3}\right)$ the

TABLE I. Regrowth rates at $550^{\circ} \mathrm{C}$.

\begin{tabular}{ccc}
\hline \hline Impurity & $\begin{array}{c}\text { Concentration } \\
(\text { atoms cm } \\
\left.\times 10^{-30}\right)\end{array}$ & $\begin{array}{c}\text { Regrowth } \\
\text { rate } \\
\left(\AA \mathrm{min}^{-1}\right)\end{array}$ \\
\hline${ }^{11} \mathrm{~B}$ & 2.5 & $1028^{\mathrm{a}}$ \\
${ }^{12} \mathrm{C}$ & 1.8 & 55 \\
${ }^{14} \mathrm{~N}$ & 2.5 & 7.7 \\
${ }^{16} \mathrm{O}$ & 2.4 & 9.0 \\
${ }^{20} \mathrm{Ne}$ & 2.8 & 3.5 \\
${ }^{28} \mathrm{Si}$ & $\ldots .55$ \\
${ }^{31} \mathrm{P}$ & 2.5 & 327 \\
${ }^{40} \mathrm{Ar}$ & 1.5 & 2.4 \\
${ }^{74} \mathrm{Ge}$ & 2.0 & 87 \\
${ }^{75} \mathrm{As}$ & 2.0 & 480 \\
\hline \hline
\end{tabular}

at Extrapolated valuc from $550^{\circ} \mathrm{C}$.

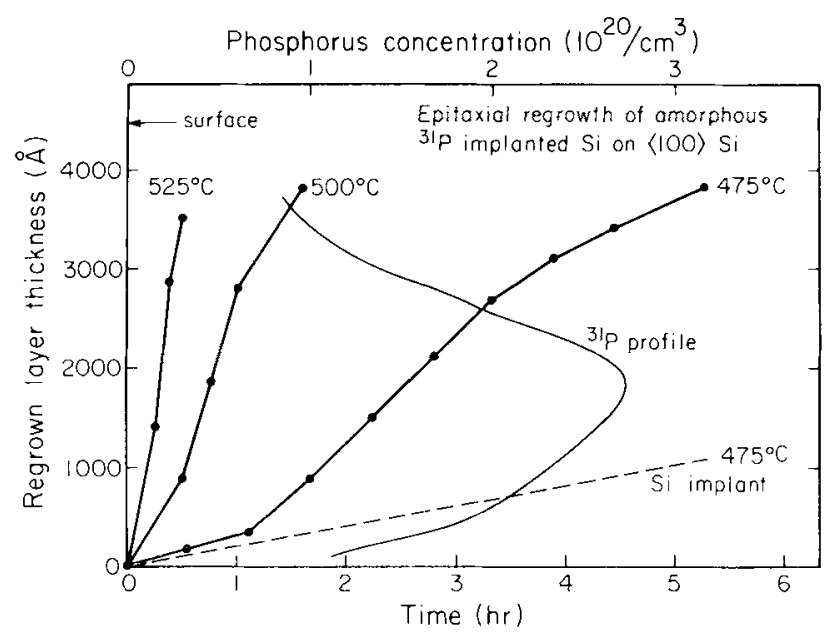

FIG. 7. Nonlinear growth rate of Si samples amorphatized by Si ion implantation and then implanted with phosphorus. Dashed line corresponds to the regrowth of ${ }^{31} \mathrm{P}$-free sample at $475^{\circ} \mathrm{C}$. This line shows the ${ }^{31} \mathrm{P}$ profile. The zero depth corresponds to the original amorphous-crystalline interface (taken from Ref. 9).

regrowth rate is only slightly faster than that for ${ }^{31} \mathrm{P}$ free samples. The rate then accelerates to a maximum as the phosphorus concentration increases to $\sim 3 \times 10^{20} \mathrm{~cm}^{-3}$.

The apparent activation energies of growth for impurity implanted layers were found to be quite similar. The activation energy for ${ }^{11} \mathrm{~B}$-implanted layers is somewhat less than and that of ${ }^{16} \mathrm{O}$-implanted layer somewhat greater than that for impurity-free Si layers.

The precise mechanisms that lead to the observed changes in growth rate are not clear at present. It is, perhaps, reasonable to speculate that some sort of cooperative reordering at the interface is present. Walser and Bené ${ }^{7}$ have analyzed the impurity effects in terms of charge transfers between impurity atoms and the $\mathrm{Si}$ atoms at the interface.

On the other hand, implanted rare gas ions, such as Ar, were found to form bubbles and grow to $\sim 200 \AA \AA$ or more in size at temperatures from $500^{\circ}$ to $600^{\circ} \mathrm{C}^{8,10,10 \mathrm{a}, 10 \mathrm{~b}}$ where regrowth occurs. Severe twinning and reduction in growth rate even below that for ${ }^{16} \mathrm{O}$-implanted samples were found in the presence of bubbles. This retardation of regrowth kinetics is, perhaps, more likely to be a physical rather than a chemical effect.

\section{Impurity redistribution due to regrowth}

The regrowth kinetics discussed thus far are applicable essentially for annealing at low temperatures $\left(\sim 500^{\circ}-650^{\circ} \mathrm{C}\right)$. At these temperatures, the Si lattice regrows usually without disturbing the as-implanted impurity profile. Significant amounts of implanted species are already in substitutional site after the low temperature regrowth. In fact, impurity substitutional concentrations in excess of the solid solubility have often been observed. It is, however, customary to anneal implanted samples at much higher temperatures $\left(\sim 900^{\circ}-\right.$ $1000^{\circ} \mathrm{C}$ ) to increase crystallinity, substitutionality and reordering rates. Under these conditions, the regrowth characteristics can have major influence on the impurity distribution following annealing. 


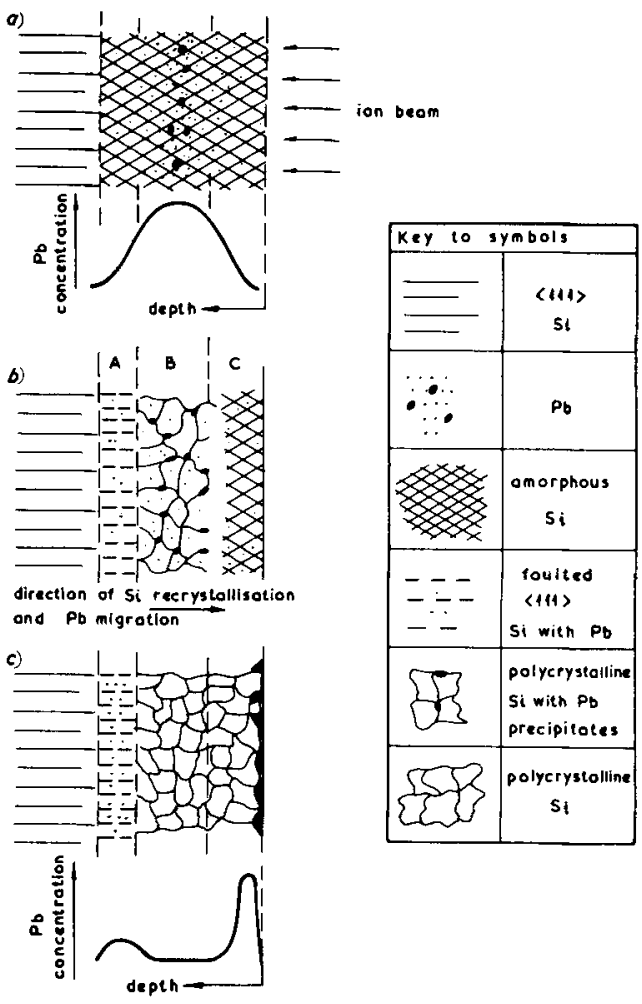

FIG. 8. Step-by-step representation of Si crystallization showing the implanted layer structure: (a) prior to reordering, (b) during reordering, and (c) after complete recrystallization (taken from Ref. 13).

The redistribution of implanted species in regrown layers has been investigated quite extensively. ${ }^{11-13}$ We will summarize the results as follows:

(1) Annealing at low temperatures $\left(\sim 550^{\circ} \mathrm{C}\right)$ of amorphous Si layers obtained by ion implantation of species such as In, $\mathrm{Sb}$, and $\mathrm{Pb}$ on $(100),(110)$, and (111) samples leads to epitaxial regrowth of these samples. Redistribution of implanted species was not observed. The crystallinity of the epitaxial layers is much superior for (100) and (110) samples than that for (111) samples.

(2) Direct annealing of these samples at high temperatures

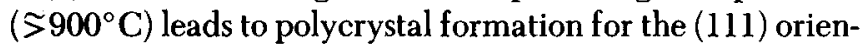
tation. Major redistribution and in some cases significant loss of implanted species are observed. For (100) and (110) samples, epitaxial regrowth occurs with varying degrees of minor redistribution and loss of implanted species depending on the implantation energy, species and the permeability of the species through a surface oxide. The redistribution of implanted species in (111) samples is consistent with the model of migration of species through the grain boundaries. Formation of polycrystalline in the (111) material at high temperatures is due to the unfavorable slow epitaxial regrowth rates compared with that for polycrystalline growth in the implanted layer. The grain boundaries, then, provide short circuit paths for the migration.

(3) Two-step annealing of (111) samples at $\sim 550^{\circ}$ and $900^{\circ} \mathrm{C}$ results in a stable impurity distribution. The amorphous layer regrows at $550^{\circ} \mathrm{C}$ to a highly defected epitaxial layer, the $900^{\circ} \mathrm{C}$ anneal reduces the residual defect clusters. The absence of high angle boundaries reduces the migration of impurities to an insignificant amount.

(4) For very high-dose implantation, there is an accumulation of impurity at the growth front. When the solubility limit of the impurity in the regrown layer is reached (this can be higher than the equilibrium solid solubility), further recrystallization results in polycrystal formation.

\section{APPLICATIONS}

The interrelationships between various parameters in the regrowth phenomena is a complex one. The simplest and most direct lesson learned from these experiments is that (111) material is more difficult to control than other orientations. On the other hand, the two step annealing cycle has found its way in many production lines in the semiconductor industry.

Recently it appears that ion implantation techniques have the potential of modifying material properties. One such example is the use of ion implantation technique to improve the crystalline quality of $\mathrm{Si}$ layers grown epitaxially on sapphire substrates (SOS). The commonly used epitaxial relationship between $\mathrm{Si}$ and sapphire is (100) Si layers epitaxially grown on (1) $1 \overline{0}$ ) sapphire substrates by chemical vapor deposition. Because of the difference in lattice constants, the interfacial region between the sapphire and $\mathrm{Si}$ is usually full of defects such as twins and dislocations. The density of defects decreases as the Si layer increases in thickness. Based on the interrelation between regrowth and orientation discussed above, it seems possible to use ion implantation techniques to create an amorphous region near the sapphire-Si interface and leave the Si top surface layer relatively damage free after implantation. One can, then, take advantage of the fact that the Si top surface layer is (100) in orientation (a fast and defect free regrowth direction), and can be used as a seed to regrow the amorphous region near the sapphire-Si interface created by implantation. The resulting Si layer after implatation and regrowth can have a much better crystalline quality than that of the original layer. This effect has actually been observed. ${ }^{14}$

On the other hand, (111) Si layer on (0001) sapphire is a less appropriate system to try ion implantation modification techniques. Since the $\langle 111\rangle$ direction is a slow and defectforming regrowth direction. However, this difficulty can be partially circumvented by implanting impurities such as As and B (regrowth accelerators) and followed by a two-step annealing cycle.

\section{CONCLUDING REMARKS}

From the discussion above, there are clearly interplays between orientation impurity, impurity distribution, regrowth kinetics and crystallinity of the regrown layer. Over the past few years, there has been more reproducible and consistent observations on the regrowth phenomena among various laboratories. A more unified picture of the regrowth processes is emerging, although a detailed understanding of the many aspects of regrowth is far from satisfaction. The advent of transient annealing techniques has stirred up an intense activity in the study of regrowth, using techniques such as laser 
and pulsed electron beam. These techniques offer fast, patterned regrowth and other advantages. The stringent clean interface requirement is also somewhat relaxed for these techniques. Under certain conditions, transient annealing causes melting of the layers. It is, then, a case of liquid phase epitaxy. Under other conditions, there is no melting involved. It is expected that transient annealing characteristics without melting follow those observed for thermal annealing. The understanding of thermal annealing behavior is, then, a bridge to the understanding of solid phase transient annealing.

\section{ACKNOWLEDGMENTS}

The financial support of the Office of Naval Research ( $\mathrm{L}$. Cooper) is gratefully acknowledged. The author also thanks J. S. Williams, J. W. Mayer, and W. L. Brown for their valuable discussions, and $\mathrm{P}$. Blood for his prompt response.

1J. A. Roth and C. L. Anderson, Appl. Phys. Lett. 31, 689 (1977).

laSee for example: S. S. Lau, W. F. Tseng, M-A. Nicolet, J. W. Mayer, R. C. Eckardt, and R. J. Wagner, Appl. Phys. Lett. 31, 33 (1978).

${ }^{2}$ See for example: S. S. Lau and W. F. van der Weg in Thin Films-Interdiffusion and Reaction, Electrochemical Monograph Series, edited by J. M. Poate, K. N. Tu, and J. W. Mayer (Wiley, New York, 1978), Chap. 12.
${ }^{3}$ L. Csepregi, E. F. Kennedy, J. W., Mayer, and T. W. Sigmon, J. Appl. Phys. (in press).

${ }^{4}$ M. D. Rechtin, P. P. Pronko, G. Foti, L. Csepregi, E. F. Kennedy, and J. W. Mayer (submitted to Phil. Mag.).

${ }^{5}$ T. Cass and T. W. Sigmon (submitted to J. Appl. Phys.).

${ }^{6} \mathrm{~K}$. Seshan and E. P. EerNisse, Appl. Phys. Lett. (to be published).

${ }^{7}$ R. M. Walser and R. W. Bené in Proceedings of the Electrochemical Society Symposium on Thin Films Phenomena-Interfaces and Interactions, edited by J. E. E. Baglin and J. M. Poate (Electrochem. Soc., Princeton, 1978), p. 284.

${ }^{8}$ E. F. Kennedy, L. Csepregi, J. W. Mayer and T. W. Sigmon, J. Appl. Phys. 48, 4241 (1977).

${ }^{9}$ L. Csepregi, E. F. Kennedy, T. J. Gallager, J. W. Mayer, and T. W. Sigmon, J. Appl. Phys. 48, 4234 (1977).

10P. Revesz, M. Wittmer, J. Roth and J. W. Mayer, (submitted to J. Appl. Phys.).

${ }^{10 a}$ A. G. Cullis, Proc. 6th Eur. Conf. Electron Microsc., Jerusalem 1, 192 (1976).

${ }^{10 b}$ A. G. Cullis, T. E. Seidel, and R. L. Meeks, J. Appl. Phys. (to be published).

${ }^{11}$ P. Blood, W. L. Brown and G. L. Miller (submitted to J. Appl. Phys.).

${ }^{12}$ P. Blood, W. L. Brown and G. L. Miller in Ion Implantation in Semiconductors, edited by F. Chernow, J. A. Borders, and D. K. Brice, (Plenum, New York, 1976).

${ }^{13}$ See for example: J. S. Williams, C. E. Christodules, W. A. Grant, R. Androw, and J. R. Brawn, Radiat. Eff. 32, 55 (1977).

${ }^{14}$ S. S. Lau, S. Matteson, J. W. Mayer, P. Revesz, J. Gyulai, T. Sigmon, and T. Cass, presented at the International Conference on Ion Beam Modification of Materials, Sept. 1978, Budapest, Hungary. 